DE-FG05-97ER41031-57

\title{
Hydrodynamics of the Vacuum
}

\author{
P. M. Stevenson \\ T. W. Bonner Laboratory, Department of Physics and Astronomy \\ Rice University, P.O. Box 1892, Houston, TX 77251-1892, USA
}

\begin{abstract}
:
Hydrodynamics is the appropriate "effective theory" for describing any fluid medium at sufficiently long length scales. This paper treats the vacuum as such a medium and derives the corresponding hydrodynamic equations. Unlike a normal medium the vacuum has no linear sound-wave regime; disturbances always "propagate" nonlinearly. For an "empty vacuum" the hydrodynamic equations are familiar ones (shallow water-wave equations) and they describe an experimentally observed phenomenon - the spreading of a clump of zero-temperature atoms into empty space. The "Higgs vacuum" case is much stranger; pressure and energy density, and hence time and space, exchange roles. The speed of sound is formally infinite, rather than zero as in the empty vacuum. Higher-derivative corrections to the vacuum hydrodynamic equations are also considered. In the empty-vacuum case the corrections are of quantum origin and the post-hydrodynamic description corresponds to the Gross-Pitaevskii equation. I conjecture the form of the post-hydrodynamic corrections in the Higgs case. In the 1+1-dimensional case the equations possess remarkable 'soliton' solutions and appear to constitute a new exactly integrable system.
\end{abstract}




\section{Introduction}

Quite generally, any fluid medium when viewed at sufficiently long length scales can be described by hydrodynamics. For length scales much, much greater than the mean free path the microscopic dynamics becomes irrelevant, except for determining the equation of state of the medium. The only relevant degrees of freedom are a density $n$ and a flow velocity $\overrightarrow{\mathbf{v}}$ : there is some 'stuff' and it flows.

The idea of this paper is very simple:- to apply hydrodynamics to the vacuum. In fact, there are two cases; (i) an "empty" vacuum, where the equilibrium density is zero, and (ii) a "spontaneous condensate," or "Higgs-type" vacuum, where the relativistic energy density is minimized for some non-zero density $n=n_{v}$. The actual physical vacuum is empty of certain particle species (e.g. electrons) but is a spontaneous condensate of others (e.g. quarks and gluons). The electroweak theory requires the vacuum to have a nonzero vacuum expectation value for the Higgs field, which one can view as a spontaneous condensate of scalar particles. This point has been well expressed by 't Hooft: "What we experience as empty space is nothing but the configuration of the Higgs field that has the lowest possible energy. If we move from field jargon to particle jargon, this means that empty space is actually filled with Higgs particles. They have Bose condensed." [1, 2]

The great virtue of a hydrodynamic approach is that it is essentially independent of the microscopic dynamics. Hydrodynamics is almost as universal and fundamental as thermodynamics and it may yield important lessons for modern particle physics. (This view is advocated from a different perspective in an important recent paper [3].) In particular, hydrodynamics may perhaps provide a route to a deeper understanding of the "Higgs-type" vacuum, a phenomenon that is a vital, but experimentally untested feature of the Standard Model.

Hydrodynamics, of course, has great limitations. It does not tell one how to produce or detect the excitations that it describes. Nor does it even tell one the scale of the phenomena, which is governed by a parameter whose value is set by the microscopic dynamics. I think it best to avoid speculations until the hydrodynamic equations and their solutions have been thoroughly explored. That task is begun in this paper, but much more remains to be done. For simplicity I concentrate on the 1+1-dimensional case, though a spherically symmetric $d+1$-dimensional solution will be discussed in Section 6 . 


\section{Hydrodynamics of a normal medium}

In this subsection I very briefly review the basics of hydrodynamics applied to a "normal," nonrelativistic medium [4, 5, 6]. I assume that the medium is "barotropic" (energy density only a function of the pressure). This is a good approximation for many normal media and will hold exactly in the vacuum case. As a further simplification I concentrate on the 1+1-dimensional case.

The hydrodynamic equations follow directly from conservation laws together with a constitutive relation for the medium, leading to coupled, first-order partial differential equations for $\rho$, the mass density, and $v$, the flow velocity. Mass conservation and momentum conservation yield the fundamental equations (subscripts indicate partial derivatives: $\rho_{t} \equiv \partial \rho / \partial t$, etc. $):$

$$
\begin{gathered}
\rho_{t}+(\rho v)_{x}=0, \\
(\rho v)_{t}+\left(\rho v^{2}+P(\rho)\right)_{x}=0,
\end{gathered}
$$

where $P(\rho)$ is the pressure as a function of density. The previous equations together imply a simpler one, known as the Euler equation:

$$
v_{t}+v v_{x}+\frac{P_{x}}{\rho}=0
$$

Provided that the solution is everywhere smooth, one may regard Eqs. (2.3) and (2.1) as the basic equations. (However, if the solution develops discontinuities it is essential to remember that Eqs. (2.1) and (2.2) are the fundamental pair.)

For a normal medium the pressure varies linearly for small density disturbances:

$$
P(\rho)=P_{\mathrm{eq}}+v_{0}^{2}(\delta \rho)+O\left((\delta \rho)^{2}\right)
$$

where $\delta \rho \equiv \rho-\rho_{\text {eq }}$ and $v_{0}^{2}$ is a constant. For sufficiently small disturbances $\left(\delta \rho \ll \rho_{\text {eq }}\right.$ and $\left.v \ll v_{0}\right)$ the hydrodynamic equations can then be linearized, yielding

$$
\begin{aligned}
& v_{t}+v_{0}^{2} \frac{(\delta \rho)_{x}}{\rho_{\mathrm{eq}}} \approx 0, \\
& (\delta)_{t}+\rho_{\mathrm{eq}} v_{x} \approx 0 .
\end{aligned}
$$

If the $x$ derivative of the first equation and the $t$ derivative of the second are combined, so as to eliminate $v$, one obtains the wave equation for $\delta \rho$ :

$$
(\delta \rho)_{t t}-v_{0}^{2}(\delta \rho)_{x x} \approx 0
$$


(Similarly, one can derive the wave equation for $v$.) Thus, in this linearized regime hydrodynamics reduces to acoustics. Disturbances propagate as sound waves and obey the superposition principle. Note that the speed of sound is determined by the thermodynamic derivative

$$
v_{0}^{2}=\left.\frac{\partial P}{\partial \rho}\right|_{\mathrm{eq}} .
$$

For larger disturbances, violating one or both of the conditions $\delta \rho \ll \rho_{\text {eq }}$, and $v \ll v_{0}$, nonlinear effects come into play. In the vacuum case, as we shall see, there is no linear, acoustic regime; disturbances are always nonlinear. Insight into the nonlinear regime can be gained by considering first the case of a pressureless fluid, where the Euler equation reduces to Burgers' equation (also known by many other names)

$$
v_{t}+v v_{x}=0
$$

The solution, easily verified by direct differentiation, can be expressed as follows [7]: Let the initial condition be $v(x, t=0)=V(x)$, then at later times

$$
v=V(\chi) \quad \text { with } \quad \chi=x-V(\chi) t,
$$

where the latter equation defines $\chi$ implicitly. Thus, velocity disturbances propagate locally at the local flow velocity. If $V$ is monotonic increasing to the right then the forward part of the wave outruns the backward part and the disturbance simply stretches out. However, if $V$ is monotonic decreasing then the backward part of the wave tends to catch up with the forward part, steepening the wave profile, as with ocean waves approaching the shore. After a finite time the solution, rather than becoming multivalued, as with waves at a beach, develops a finite discontinuity in $v$, a shock wave.

Quite generally, the solution may develop shocks (finite discontinuities in $\rho$ and/or $v$ ) in finite time, even from very smooth initial conditions. Requiring mass and momentum conservation yields two equations:

$$
\text { shock speed }=\frac{[[\rho v]]}{[[\rho]]}=\frac{\left[\left[\rho v^{2}+P(\rho)\right]\right]}{[[\rho v]]},
$$

where $[[X]] \equiv X_{\text {right }}-X_{\text {left }}$. The shock solution, even though the first derivatives do not exist at the shock, can be regarded as a "weak solution," a solution in a distributiontheoretic sense, of the original equations [8]. An important warning here is that the mathematical notion of a "weak solution" must be applied to the fundamental mass and momentum conservation equations, (2.1) and (2.2), and not to the Euler equation (2.3). 
At a shock the solution violates the basic hydrodynamic assumption that all relevant length scales are much greater than the mean free path. Thus, corrections to hydrodynamics play a role. These corrections can be developed as a series expansion in (mean free path)/(length scale), known as the Chapman-Enskog expansion [9]. For a normal medium the post-hydrodynamic corrections involve dissipative effects such as viscosity. The correction terms involve second derivatives and violate (macroscopic) time-reversal invariance, since they entail entropy generation. In the pressureless-fluid case one encounters the viscous Burgers' equation [10].

$$
v_{t}+v v_{x}=\epsilon v_{x x}
$$

in which the viscosity term $\epsilon v_{x x}$ smooths out the shock discontinuity. For this equation, and more generally, one may find the shock profile by looking for "travelling wave" solutions with $v=v(x-a t)$ and $\rho=\rho(x-a t)$ where $a=$ is a constant and $v_{L} \neq v_{R}$ and/or $\rho_{L} \neq \rho_{R}$, where $v_{L} \equiv v(x \rightarrow-\infty)$, etc.. As $\epsilon \rightarrow 0$ the travelling wave's profile narrows, approaching a discontinuity, and the speed $a$ tends to the shock speed of Eq. (2.11). In this sense, a hydrodynamic solution with shocks is still a meaningful approximate description of the physics.

\section{Constitutive relation of the vacuum medium}

Henceforth a relativistic framework will be adopted; energy and energy density will include the rest-energy $\left(m c^{2}\right)$ contribution and the speed of light, $c$, will be set to 1 .

The "empty vacuum" and "Higgs vacuum" cases are illustrated in Figs. 1 and 2. In both cases, since we are dealing with a zero-temperature medium, the pressure is given by

$$
P=-\mathcal{E}+n \frac{d \mathcal{E}}{d n}
$$

where $\mathcal{E}$ is the energy density and $n$ is the number density of particles. Note that a precise definition of $n$ is not needed. What matters physically is the "constitutive relation" how $P$ varies with $\mathcal{E}$ in the vicinity of the equilibrium state, and it is merely a convenience to express this relation parametrically as $\mathcal{E}=\mathcal{E}(n)$ and $P=P(n)$ via the above equation. If, for example, the underlying microscopic theory is a scalar quantum field theory, one may take $n$ to be proportional to $\phi^{2}$, where $\phi$ is the classical field, and identify $\mathcal{E}(n)$ with the field-theoretic effective potential $V_{\text {eff }}(\phi)$.

The empty-vacuum case is essentially nonrelativistic. The energy density $\mathcal{E}$ is dominated by the mass density (times $c^{2}$, but $c=1$ here). However, this linear term exactly 


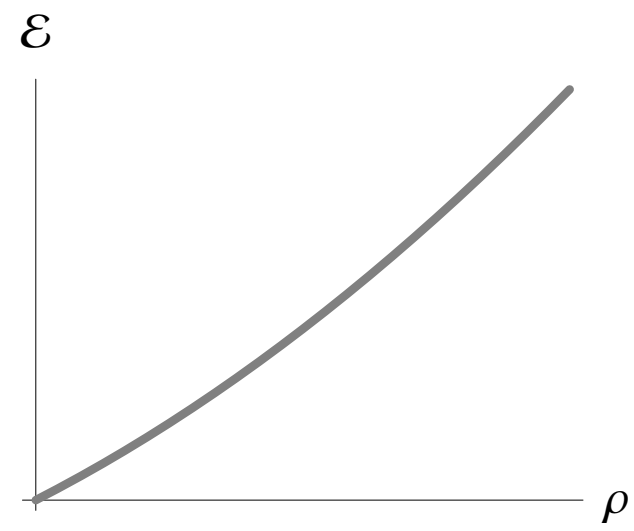

Figure 1: The empty vacuum case; energy density as a function of mass density $\rho=m n$. The equilibrium density is $\rho=0$.

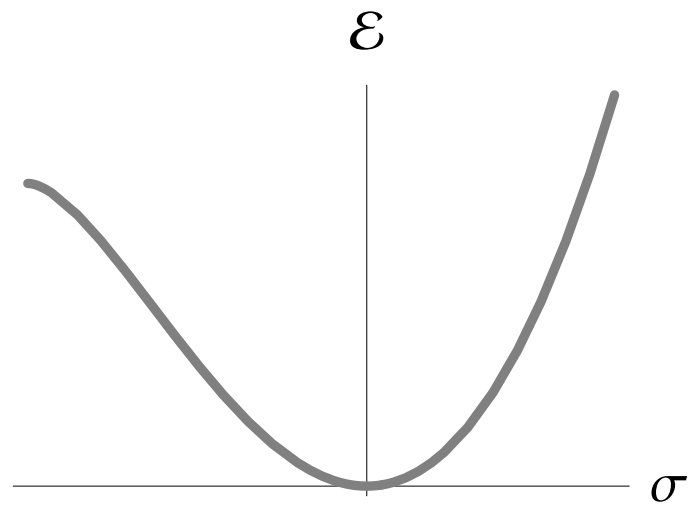

Figure 2: The Higgs vacuum case; energy density as a function of $\sigma=\frac{1}{C}\left(\frac{n}{n_{v}}-1\right)$ where $\mathcal{C}$ is a constant. 
cancels in the pressure (3.13), so that the small non-linear term $\frac{1}{2} g \rho^{2}$ is crucial [1]:

$$
\begin{gathered}
\mathcal{E}=\rho+\frac{1}{2} g \rho^{2}+\ldots, \\
P=\frac{1}{2} g \rho^{2}+\ldots
\end{gathered}
$$

The speed of sound is formally given by

$$
\left.v_{0}^{2} \equiv \frac{\partial P}{\partial \mathcal{E}}\right|_{\rho=0}=\left.\frac{d P}{d \rho}\right|_{\rho=0}=0 .
$$

In the Higgs-vacuum case we find a "mirror-image" situation with the forms of $\mathcal{E}$ and $P$ interchanged. (For convenience I subtract a constant from the energy density so that $\mathcal{E}$ vanishes in equilibrium. Since gravity is not being included in the discussion this step is quite innocuous.)

$$
\begin{gathered}
\mathcal{E}=\frac{1}{2} C \sigma^{2}+\ldots, \\
P=\sigma+\frac{1}{2} C \sigma^{2}+\ldots,
\end{gathered}
$$

with [12]

$$
\sigma=\frac{1}{C}\left(\frac{n}{n_{v}}-1\right)
$$

where $C$ is a positive constant. $C$ is the compressibility of the vacuum medium

$$
C=\left(\left.n_{v}^{2} \frac{d^{2} \mathcal{E}}{d n^{2}}\right|_{n=n_{v}}\right)^{-1} .
$$

For small disturbances one has $P \gg \mathcal{E}$, so the Higgs-vacuum case is ultrarelativistic. The speed of sound is formally infinite [14:

$$
\left.v_{0}^{2} \equiv \frac{\partial P}{\partial \mathcal{E}}\right|_{\sigma=0}=\left.\frac{d P / d \sigma}{d \mathcal{E} / d \sigma}\right|_{\sigma=0}=\frac{1}{0}=\infty .
$$

This is the first sign that something very interesting — not to say bizarre — is going on. Note that the vanishing denominator arises because $d \mathcal{E} / d n$ vanishes at $n_{v}$, which is the defining property of a Higgs-type vacuum.

To anticipate (and oversimplify), the empty-vacuum case behaves like Burgers equation $v_{t}+v v_{x}=0$ and effects tend to "propagate" at the local flow velocity $v$, while the Higgsvacuum case behaves like the $x \leftrightarrow t$ Burgers equation $v_{t}+\frac{1}{v} v_{x}=0$ whose implicit solution $v=v\left(x-\frac{1}{v} t\right)$ implies that disturbances tend to "propagate" at speed $\frac{1}{v}$ (that is $c^{2} / v$, restoring $c$ ). Thus, the "propagation" speed is superluminal, and can be arbitrarily large as $v$ becomes arbitrarily small. This is a fundamental and inescapable characteristic of 
the Higgs-type vacuum. Obviously, deep issues related to causality are involved. Note that it is well known that an ultrarelativistic medium (where the pressure is much greater than the energy density) can have a superluminal speed of sound [15]. Discussions of the causality issue for an ultrarelativistic medium can be found in Ref. [16, 17. I set this issue aside for the present.

\section{Derivation of the vacuum hydrodynamic equations}

The energy-momentum tensor for a perfect fluid has the form $\left[g^{\mu \nu}=\operatorname{diag}(1,-1,-1,-1)\right]$

$$
T^{\mu \nu}=(\mathcal{E}+P) u^{\mu} u^{\nu}-P g^{\mu \nu},
$$

where $\mathcal{E}$ and $P$ are respectively the energy density and the pressure in the co-moving frame, and $u^{\mu}$ is the flow 4 -velocity

$$
u^{\mu}=\left(u_{0}, \vec{u}\right)=(\gamma, \gamma \overrightarrow{\mathbf{v}})
$$

with $\gamma \equiv 1 / \sqrt{1-v^{2}}$. Energy and momentum conservation equations follow from $\partial_{\mu} T^{\mu \nu}=$ 0 with, respectively, $\nu=0$ and $\nu=i=1,2,3$ :

$$
\begin{gathered}
\frac{\partial}{\partial t}\left[(\mathcal{E}+P) u_{0}^{2}-P\right]+\frac{\partial}{\partial x^{j}}\left[(\mathcal{E}+P) u^{j} u_{0}\right]=0, \\
\frac{\partial}{\partial t}\left[(\mathcal{E}+P) u_{0} u^{i}\right]+\frac{\partial}{\partial x^{j}}\left[(\mathcal{E}+P) u^{j} u^{i}+P \delta^{i j}\right]=0 .
\end{gathered}
$$

Substituting for $\mathcal{E}$ and $P$ - from either (3.14, 3.15) in the empty-vacuum case, or 3.17 3.18) in the Higgs-vacuum case - yields the fundamental equations of "vacuum hydrodynamics."

Provided the solution is free of shock discontinuities and singularities such as vortex lines, one may work with equivalent equations that are somewhat neater. (Cf. the discussion below Eq. (2.3).) These neater equations arise from $u_{\nu} \partial_{\mu} T^{\mu \nu}=0$ ("energy conservation in the co-moving frame") and $\left(g_{\alpha \nu}-u_{\alpha} u_{\nu}\right) \partial_{\mu} T^{\mu \nu}=0$ ("Euler equation") [18. In the empty vacuum case (in $1+1$ dimensions) these equations are:

$$
\begin{gathered}
(\gamma \rho)_{t}+(\gamma \rho v)_{x}=0 \\
((1+g \rho) \gamma v)_{t}+((1+g \rho) \gamma)_{x}=0
\end{gathered}
$$

Note that one could re-scale $\rho$ to $\hat{\rho}=g \rho$ and hence eliminate $g$ from the equations.

Since one is interested in small disturbances it is natural to assume that $v$ and $g \rho$ are small. I shall refer to this as the nonrelativistic-flow approximation (NFA). In the 
empty-vacuum case it is equivalent to nonrelativistic reduction. Restoring the factors of $c$ and considering $1 / c$ to be a small parameter, $\epsilon$, one obtains the bookkeeping rules for the NFA [20]

$$
v=O(\epsilon), \quad g \rho=O\left(\epsilon^{2}\right), \quad \frac{\partial}{\partial t}=O(\epsilon) .
$$

Applying these rules to the above equations yields

$$
\begin{gathered}
\rho_{t}+(\rho v)_{x}=0, \\
v_{t}+\left(\frac{1}{2} v^{2}+g \rho\right)_{x}=0 .
\end{gathered}
$$

Comparing these equations with (2.1), (2.3), one sees that they correspond to an otherwise "normal" medium with a pressure function $P(\rho)=\frac{1}{2} g \rho^{2}$. This is a special case of a "polytropic gas" $\left(P \propto \rho^{n}\right)$ with polytropic index $n=2$ [4, 5, 6, 19]. Ordinary gases are, to a good approximation, polytropic, but with $n$ between 1 and $5 / 3$. However, the $n=2$ case arises in the treatment of shallow water waves (wavelengths long compared to the depth of the water), where $\rho$ is proportional to the vertical displacement of the water surface [5].

More relevantly, these equations also arise in the description of the free expansion of an atomic Bose-Einstein condensate when the trapping potential is turned off [21, 22. This fact is natural from the present point of view: the clump of atoms, localized with a particular density distribution at $t=0$, is just a particular disturbance of the empty vacuum. Provided that the initial density distribution is sufficiently slowly varying, its spreading will naturally be governed by the vacuum hydrodynamic equations. This important example is discussed in more detail in Section 6.

There is an important conceptual subtlety with the NFA. The original equations are Lorentz invariant, while the NFA equations are only Galilean invariant (i.e., only approximately Lorentz invariant for small boosts). Thus, using the NFA implies restricting oneself to a particular Lorentz frame and its "neighbouring" frames. For a normal medium the rest frame of the medium obviously plays this role. However, the vacuum is a Lorentzinvariant medium; it has no rest frame. The appropriate frame for the NFA is determined solely by the initial conditions. If in some frame the NFA conditions are satisfied at $t=0$ then they will remain satisfied at all later times. One may trivially take a NFA solution and boost it by a large Lorentz boost to obtain an approximate solution to the original relativistic equations in which $v$ is everywhere close to 1 . Only when the range of $v$ values is a significant fraction of unity is it necessary to abandon the NFA and return to the relativistic equations, (4.26, 4.27). 
In the Higgs vacuum case the pressure and the energy density exchange roles. As a consequence, time and space in some sense exchange roles. In the $1+1$ dimensional case one obtains

$$
\begin{gathered}
(\gamma \sigma)_{x}+(\gamma \sigma v)_{t}=0 \\
((1+C \sigma) \gamma v)_{x}+((1+C \sigma) \gamma)_{t}=0
\end{gathered}
$$

which are the same as the empty vacuum case with $x \leftrightarrow t$ (and $\rho \rightarrow \sigma, g \rightarrow C$ ). (In higher dimensions the "space $\leftrightarrow$ time" interchange is obviously more complicated. See Eqs. (6.46, 6.47) below.)

Using this symmetry one can define a consistent NFA by the bookkeeping rules:

$$
v=O(\epsilon), \quad C \sigma=O\left(\epsilon^{2}\right), \quad \frac{\partial}{\partial x}=O(\epsilon) .
$$

and obtain the equations

$$
\begin{gathered}
\sigma_{x}+(\sigma v)_{t}=0, \\
v_{x}+\left(\frac{1}{2} v^{2}+C \sigma\right)_{t}=0 .
\end{gathered}
$$

Although the flow velocity is nonrelativistic $(v \ll 1)$, disturbances tend to "propagate" superluminally, at $1 / v$. Hence, the NFA here is not a normal nonrelativistic reduction. The resulting equations are "anti-Galilean" invariant. Consider two Lorentz frames, with the primed frame moving at velocity $w$ with respect to the unprimed frame. If $w$ is small, $O(\epsilon)$, then the Lorentz transformations become approximately $\left(\gamma_{w} \equiv 1 / \sqrt{1-w^{2}}\right)$

$$
\begin{gathered}
\frac{\partial}{\partial t^{\prime}}=\gamma_{w}\left(\frac{\partial}{\partial t}+w \frac{\partial}{\partial x}\right) \approx \frac{\partial}{\partial t} . \\
\frac{\partial}{\partial x^{\prime}}=\gamma_{w}\left(\frac{\partial}{\partial x}+w \frac{\partial}{\partial t}\right) \approx \frac{\partial}{\partial x}+w \frac{\partial}{\partial t} .
\end{gathered}
$$

This is backwards with respect to the usual Galilean transformation, where the $x$ derivative would not change and the $t$ derivative would change by a $w$ term.

This is certainly strange, and takes some getting used to, but one should simply view it as an approximation to the full Lorentz transformations, valid in the stated context. One is used to dealing with small objects that move slowly, so that their density distributions vary rapidly in space, but slowly in time. In the present case one is dealing with large objects, slowly varying in space, but relatively rapidly varying in time. This is related to the fact that the Higgs vacuum, as a spontaneous Bose-Einstein condensate, has almost all its particles in the same quantum state. Small disturbances of this state involve vast numbers of particles, spread over long distances, all moving nearly in lockstep, so that the disturbance varies only slowly with position while the whole collective has the same, relatively rapid time dependence. 


\section{Shock speeds}

Returning to the fundamental, relativistic energy and momentum conservation equations one can write down the two equations governing shock discontinuities. In the emptyvacuum case one finds

$$
\text { shock speed }=\frac{\left[\left[\rho(1+g \rho) \gamma^{2} v\right]\right]}{\left[\left[\left(\rho(1+g \rho) \gamma^{2}-\frac{1}{2} g \rho^{2}\right]\right]\right.}=\frac{\left[\left[\rho(1+g \rho) \gamma^{2} v^{2}+\frac{1}{2} g \rho^{2}\right]\right]}{\left[\left[\rho(1+g \rho) \gamma^{2} v\right]\right]},
$$

which in the NFA indeed reduces to the usual, nonrelativistic result (2.11) with $P(\rho)=$ $\frac{1}{2} g \rho^{2}$.

In the Higgs-vacuum case the corresponding equations are

$$
\text { shock speed }=\frac{\left[\left[\sigma(1+C \sigma) \gamma^{2} v\right]\right]}{\left[\left[\sigma(1+C \sigma) \gamma^{2}-\sigma\left(1+\frac{1}{2} C \sigma\right)\right]\right]}=\frac{\left[\left[\sigma(1+C \sigma) \gamma^{2} v^{2}+\sigma\left(1+\frac{1}{2} C \sigma\right)\right]\right]}{\left[\left[\sigma(1+C \sigma) \gamma^{2} v\right]\right]} .
$$

Since $\gamma^{2} v^{2}=\gamma^{2}-1$, one may re-write these equations as

$$
\text { shock speed }=\frac{\left[\left[\sigma(1+C \sigma) \gamma^{2} v\right]\right]}{\left[\left[\sigma(1+C \sigma) \gamma^{2} v^{2}+\frac{1}{2} C \sigma^{2}\right]\right]}=\frac{\left[\left[\sigma(1+C \sigma) \gamma^{2}-\frac{1}{2} C \sigma^{2}\right]\right]}{\left[\left[\sigma(1+C \sigma) \gamma^{2} v\right]\right]} .
$$

Comparing with (5.38) one finds that the shock speeds in the two cases are reciprocals of each other, in keeping with the $x \leftrightarrow t$ symmetry. In the NFA the equations reduce to

$$
\text { shock speed }=\frac{[[\sigma v]]}{\left[\left[\sigma v^{2}+\frac{1}{2} C \sigma^{2}\right]\right]}=\frac{[[\sigma]]}{[[\sigma v]]} \text {. }
$$

\section{An exact solution}

In $1+1$ dimensions, the empty-vacuum hydrodynamic equations in the NFA, 4.29, 4.30), are the well-known "shallow-water-wave equations," a special case of the polytropic gas. Thus, a general, exact 1+1-dimensional solution can, in principle, be obtained using the method described in Sect. 98 of Ref. [4. I shall not pursue that program here, but rather I shall discuss a specific $1+1$ dimensional solution that has a clear physical interpretation in terms of the free expansion of an atomic Bose-Einstein condensate - a phenomenon has been studied both theoretically [22] and experimentally [23]. From this solution, by $x \leftrightarrow t$, one can then obtain a solution to the 1+1-dimensional Higgs-vacuum hydrodynamic equations; a solution that can then be generalized to a spherically symmetric solution in $d+1$ dimensions.

Ref. 22] has found an exact, analytic solution of the empty-vacuum hydrodynamic equations in arbitrary dimensions. Let us consider that solution in $1+1$ dimensions [24]:

$$
\left.\begin{array}{c}
g \rho=\frac{\mathcal{N}^{2}}{4} \frac{1}{b^{3}}\left(b^{2}-\frac{x^{2}}{\ell^{2}}\right), \\
v=\mathcal{N} \sqrt{\frac{b-1}{b^{3}}} \frac{x}{\ell},
\end{array}\right\} \quad-b \ell<x<b \ell,
$$


where $\mathcal{N}$ is a normalization constant and $b$ is a function of $t$ given by

$$
\sqrt{b(b-1)}+\ln (\sqrt{b}+\sqrt{b-1})=\frac{\mathcal{N}}{\ell} t .
$$

It is straightforward to show that $b$ is a solution to the differential equations

$$
\frac{d b}{d t}=\frac{\mathcal{N}}{\ell} \sqrt{\frac{b-1}{b}}
$$

and

$$
\frac{d^{2} b}{d t^{2}}=\frac{1}{2 b^{2}} \frac{\mathcal{N}^{2}}{\ell^{2}}
$$

and hence to verify that Eqs. (4.29, 4.30) are exactly satisfied. Note that the normalization constant $\mathcal{N}$ should be small and that the scaling of $v, g \rho, \partial / \partial t$ with $\mathcal{N}$ is then completely consistent with the requirements of the NFA, Eq. (4.28).

The function $b(t)$ starts from $b=1$ at $t=0$ and grows linearly for large $t$. At $t=0$ the initial flow velocity is zero everywhere and the initial density distribution is an inverted parabola between $x=-\ell$ and $x=+\ell$ and zero outside. The solution is self-similar, in that the density maintains an inverted parabolic form as its spreads out. See Fig. 3.

The initial density distribution is in fact the equilibrium density distribution in a harmonic trapping potential, in the Thomas-Fermi approximation. Thus, the solution with these initial conditions is naturally realized in atom-trap experiments, simply by abruptly turning off a pre-existing trapping potential [22, 23].

In Fig. 3. I have taken $v=0$ outside the range $-b \ell<x<+b \ell$. This choice is somewhat arbitrary because, of course, $v$ is physically meaningless anywhere where $\rho=0$. The fundamental equations are mass and momentum conservation, (2.1) and (2.2) with $P=\frac{1}{2} g \rho^{2}$, which are identically satisfied if $\rho=0$, whatever $v$ may be doing. The discontinuity in the derivative of $\rho$ at $x= \pm b \ell$, whether or not one wishes to regard it as a "shock," is certainly a place where the hydrodynamic assumption of slow variation breaks down. Corrections to hydrodynamics come into play to smooth out these "corners" in the density distribution [25]. I discuss these corrections in the next section.

By making the transformation $x \leftrightarrow t$ one can obtain an exact solution to the Higgsvacuum hydrodynamic equations. First note that the solution in Fig. 3 can be joined smoothly on to its time-reversed solution at negative times. One can then take slices of Fig. 3 at various values of $x$, which becomes the new $t$. This leads to Fig. 4 which shows the time evolution of a $\sigma$ disturbance initially concentrated around $x=0$, though with long tails out to infinity on each side, and with $v=0$ everywhere. The disturbance spreads initially somewhat like the empty vacuum case, but then develops a dip at the centre; it 

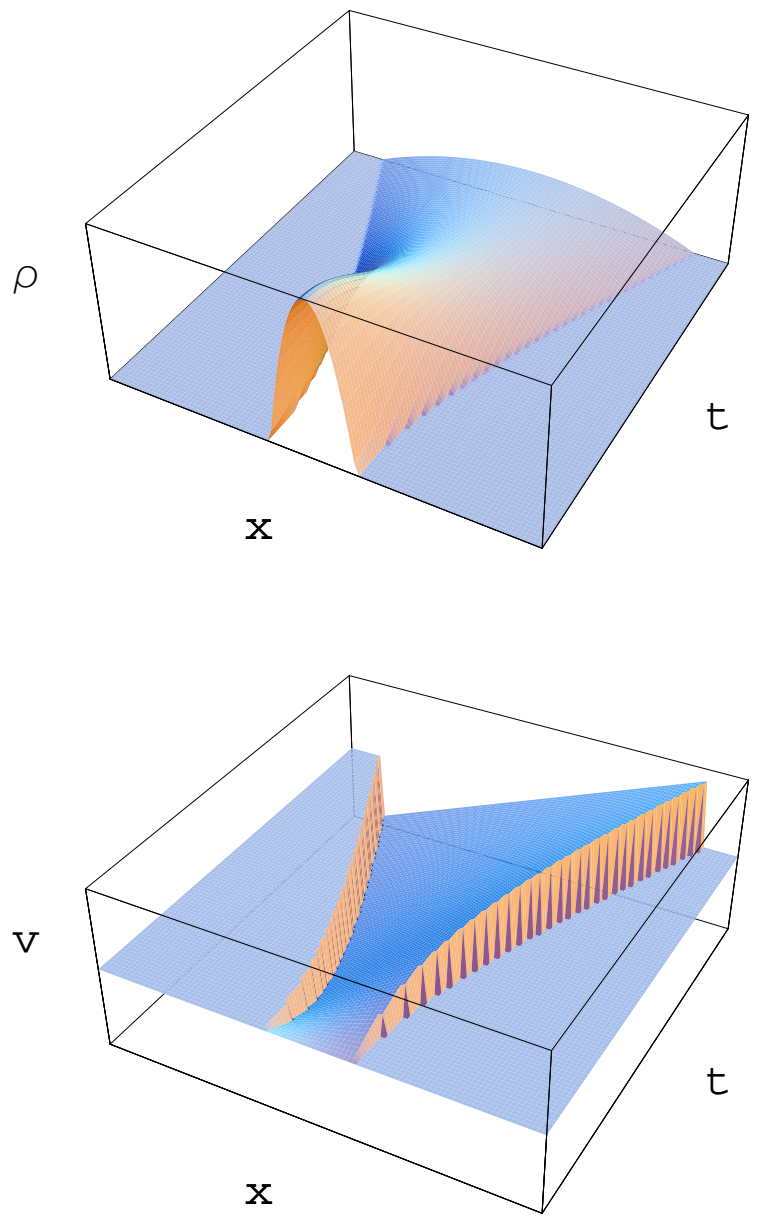

Figure 3: Density and flow velocity as functions of $x$ and $t$ for the solution in Eq. [6.42). 
then splits into two disturbances moving rapidly (superluminally) apart from each other, with each one spreading out. Between them is a stretch of perfectly restored vacuum, $\sigma=0$.

This solution can be generalized to $d+1$ dimensions. The vacuum hydrodynamic equations (4.34, 4.35) generalize to

$$
\begin{gathered}
\vec{\nabla} \sigma+(\sigma \overrightarrow{\mathbf{v}})_{t}=0, \\
v v_{t}+\vec{\nabla} \cdot \overrightarrow{\mathbf{v}}+\sigma_{t}=0 .
\end{gathered}
$$

Assuming spherical symmetry, so that $\overrightarrow{\mathbf{v}}$ is radial, gives

$$
\begin{gathered}
\sigma_{r}+(\sigma v)_{t}=0, \\
v_{r}+(d-1) \frac{v}{r}+\left(\frac{1}{2} v^{2}+C \sigma\right)_{t}=0 .
\end{gathered}
$$

Note that the extra term, with respect to the $1+1$ dimensional case, occurs in the second, not the first, equation. One may now proceed to look for a self-similar solution of the form

$$
\left.\begin{array}{c}
C \sigma=\frac{\mathcal{N}^{2}}{4} \frac{1}{b^{3}}\left(b^{2}-\frac{t^{2}}{\tau^{2}}\right), \\
v=\mathcal{N} f \frac{t}{\tau},
\end{array}\right\} \quad-b \tau<t<b \tau,
$$

where $b$ and $f$ are functions only of $\hat{r} \equiv \frac{\mathcal{N}}{\tau} r$. Substituting this Ansatz in the first equation produces a solution if

$$
f=\frac{b^{\prime}}{b}
$$

where $b^{\prime} \equiv \frac{d b}{d \hat{r}}$. Then the second equation reduces to an ordinary differential equation for $b(\hat{r}):$

$$
b^{\prime \prime}+(d-1) \frac{b^{\prime}}{\hat{r}}-\frac{1}{2} \frac{1}{b^{2}}=0 .
$$

In the $1+1$ dimensional case this equation, multiplied through by $b^{\prime}$, integrates up immediately to yield (6.43) with $t \rightarrow r$ and $\ell \rightarrow \tau$. In higher dimensions the equation is less tractable, analytically. However, it is easy to find a special, power-law solution valid for $d>\frac{4}{3}:$

$$
b_{0}=\left(\frac{3}{4} \frac{1}{\left(d-\frac{4}{3}\right)}\right)^{1 / 3} \hat{r}^{2 / 3} .
$$

With numerical methods one can find solutions that start from $b=1$ at $\hat{r}=0$ and approach this behaviour at large $\hat{r}$. These solutions provide the analog to the $1+1$ dimensional solution discussed earlier, except that the large- $r$ dependence is $r^{2 / 3}$ rather than linear. The small- $\hat{r}$ behaviour is

$$
b=1+\frac{1}{4 d} \hat{r}^{2}+O\left(\hat{r}^{4}\right) .
$$



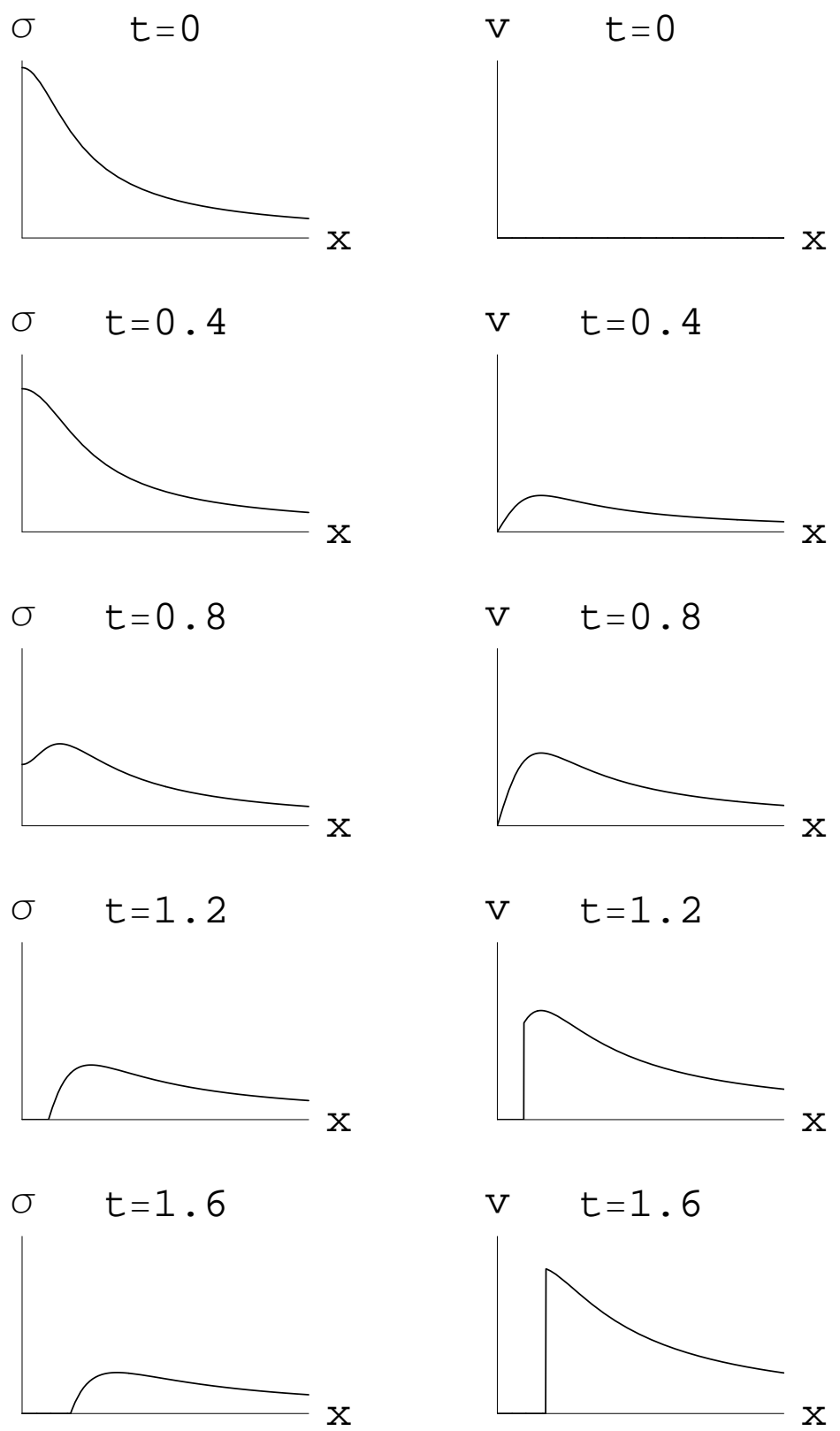

Figure 4: Solution to the 1-dimensional Higgs-vacuum hydrodynamic equations obtained by $x \leftrightarrow t$ from Fig. 3. Only positive $x$ is shown; $\sigma$ is symmetric and $v$ is antisymmetric in $x$. Time is in units of $\ell / \mathcal{N}$. A qualitatively similar spherically symmetric solution exists in higher dimensions. 
The subleading behaviour at large- $\hat{r}$ is rather curious. Writing $b=b_{0}+\beta$ and assuming $\beta \ll b_{0}$ yields a linear equation for $\beta$ :

$$
\beta^{\prime \prime}+(d-1) \frac{\beta^{\prime}}{\hat{r}}+\frac{4}{3}\left(d-\frac{4}{3}\right) \frac{\beta}{\hat{r}^{2}}=0 .
$$

Putting $\hat{r}=e^{z}$ yields an equation with constant coefficients:

$$
\ddot{\beta}+(d-2) \dot{\beta}+\frac{4}{3}\left(d-\frac{4}{3}\right) \beta=0,
$$

with solutions $\beta=e^{p z}$ with

$$
p=\frac{1}{6}\left(6-3 d \pm \sqrt{100-84 d+9 d^{2}}\right) .
$$

For $d=8$ and above the roots are real, but for $d=2, \ldots 7$ the roots are complex. For $d=3$ one finds

$$
\beta=\frac{c_{1}}{\sqrt{r}} \cos \left(\frac{\sqrt{71}}{6} \ln r+c_{2}\right)
$$

where $c_{1}, c_{2}$ are constants. The presence of the oscillatory factor is, however, all but invisible in a plot of $b(\hat{r})$. See Fig. 5 . Since $b$ is qualitatively similar to the $1+1$ dimensional case (the main difference being the $r^{2 / 3}$ rather than linear growth at large $r$ ) the solution for $\sigma$ and $v$ as a function of radius at various times is qualitatively similar to Fig. 4.

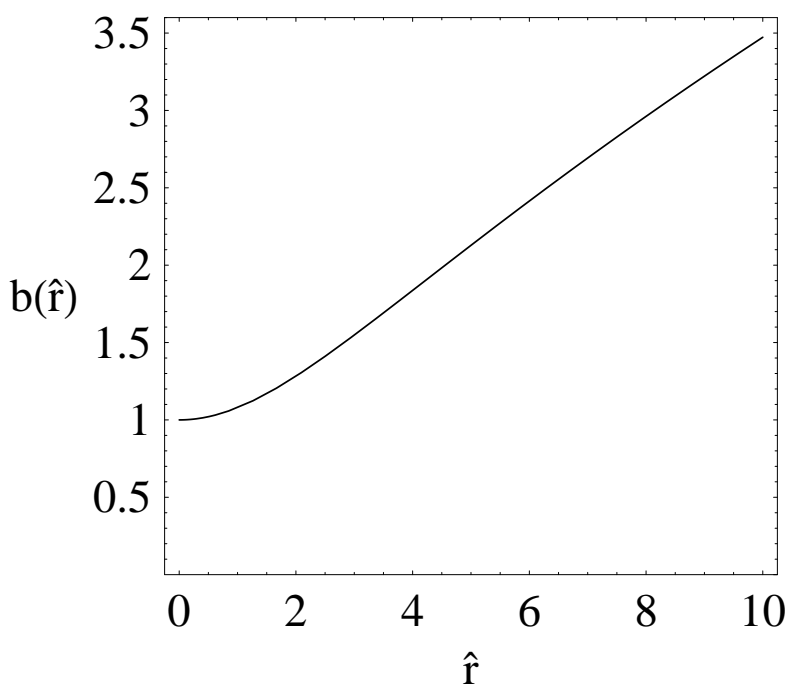

Figure 5: The solution to Eq. (6.52) with $d=3$, for $b(0)=1, b^{\prime}=0 .(\hat{r}=(\mathcal{N} / \tau) r)$. 


\section{Corrections to empty-vacuum hydrodynamics}

In the atomic Bose-Einstein condensate literature the empty-vacuum hydrodynamic equations arise as an approximation to the Gross-Pitaevskii (GP) equation: 25]

$$
i \hbar \frac{\partial \psi}{\partial t}=-\frac{\hbar^{2}}{2 m} \nabla^{2} \psi+V(\mathbf{r}) \psi+U_{0}|\psi|^{2} \psi
$$

The constant $U_{0}=4 \pi a \hbar^{2} / m$, where $a$ is the $s$-wave scattering length is related to the constant $g$ introduced earlier by $g=U_{0} / m^{2}$. The GP equation itself is valid provided the medium is dilute; $n a^{3} \ll 1$. In the present context there is no external potential, so $V(\mathbf{r})=0$, and for simplicity I consider only the $1+1$ dimensional case.

Substituting

$$
\psi=\sqrt{\frac{\rho}{m}} e^{i \varphi}, \quad \text { with } \quad v=\frac{\hbar}{m} \varphi_{x}
$$

and separating real and imaginary parts, one obtains two equations [25, 26]. One is mass conservation:

$$
\rho_{t}+(\rho v)_{x}=0
$$

and the other, after taking $\partial / \partial x$, becomes

$$
v_{t}+\left(\frac{1}{2} v^{2}+g \rho-\mathcal{A}\left(\frac{\rho_{x x}}{\rho}-\frac{1}{2} \frac{\rho_{x}^{2}}{\rho^{2}}\right)\right)_{x}=0,
$$

with $\mathcal{A}=\frac{1}{4} \frac{\hbar^{2}}{m^{2}}$. These two equations reduce to the hydrodynamic ones when the density is so slowly varying that one may neglect the "quantum pressure" term proportional to $\mathcal{A}$.

Note that the corrections to empty-vacuum hydrodynamics involve third derivatives, rather than the second derivative terms characteristic of viscosity. The corrections are non-dissipative and preserve the time-reversal $(t \rightarrow-t, v \rightarrow-v)$ and parity $(x \rightarrow-x$, $v \rightarrow-v)$ symmetries of the hydrodynamic equations.

The two real equations (7.61) and (17.62) are equivalent to the single complex GP equation. It is perhaps helpful to think of this system of equations as the senior member of a family of four equation systems, the others corresponding special cases with $g$ or $\mathcal{A}$ or both set to zero; see Table 1 . With $g=\mathcal{A}=0$ we have the Burgers system, corresponding to a pressureless gas of free, classical particles. This system has been studied recently by Choquard [27, who points out the solution $\rho \propto v_{x}$ [28]. Inclusion of particle interactions (non-zero $g$ ) leads to the empty-vacuum hydrodynamic equations. Returning to free particles $(g=0)$ but including the quantum term (non-zero $\mathcal{A}$ ) corresponds to the free 


\begin{tabular}{|l|l|}
\hline Burgers & Empty vacuum hydrodynamics \\
$v_{t}+\left(\frac{1}{2} v^{2}\right)_{x}=0$ & $v_{t}+\left(\frac{1}{2} v^{2}+g \rho\right)_{x}=0$ \\
$\rho_{t}+(\rho v)_{x}=0$ & $\rho_{t}+(\rho v)_{x}=0$ \\
\hline Schrödinger & Gross-Pitaevskii \\
$v_{t}+\left(\frac{1}{2} v^{2}-\mathcal{A}\left(\frac{\rho_{x x}}{\rho}-\frac{1}{2} \frac{\rho_{x}^{2}}{\rho^{2}}\right)\right)_{x}=0$ & $v_{t}+\left(\frac{1}{2} v^{2}+g \rho-\mathcal{A}\left(\frac{\rho_{x x}}{\rho}-\frac{1}{2} \frac{\rho_{x}^{2}}{\rho^{2}}\right)\right)_{x}=0$ \\
$\rho_{t}+(\rho v)_{x}=0$ & $\rho_{t}+(\rho v)_{x}=0$ \\
\hline
\end{tabular}

Table 1: Family of four equation systems related to empty-vacuum hydrodynamics. $\mathcal{A} \equiv$ $\hbar^{2} /\left(4 m^{2}\right)$.

Schrödinger equation, which is manifestly linear in its usual complex form. With both $g$ and $\mathcal{A}$ non-zero one has the nonlinear GP equation. Appendix A discusses the infinite tower of conservation laws associated with these equation systems.

\section{Corrections to Higgs vacuum hydrodynamics}

What are the appropriate higher-derivative corrections to the hydrodynamic equations in the Higgs-vacuum case? To answer this question, in the absence of a microscopic theory, necessarily involves some guesswork. Nevertheless some simple physical considerations, together with a mathematical/aesthetic criterion appear to point to a unique choice.

The physical considerations from the analogy with the empty vacuum case are (i) one expects third-derivative, non-dissipative, time-reversal invariant terms; (ii) in the NFA these will be time derivatives because space derivatives count as $O(\epsilon)$, (iii) the new constant multiplying the new terms should count as $O\left(\epsilon^{2}\right)$. However, there is a further physical consideration that suggests that the new terms should break the $x \leftrightarrow t$ relation to the empty vacuum case: unlike the empty-vacuum case, $v$ is meaningful even when $\sigma=0$. That is because in the Higgs case there is a pre-existing density of particles: a disturbance that causes these particles to move non-uniformly will cost energy, even if it nowhere changes the density at some initial instant.

Guided by these considerations and a mathematical/aesthetic criterion that the new equations, in $1+1$ dimensions, should be an integrable system, I looked for equation systems

that would generate a tower of conservation laws. In this way I was led to the following equations:

$$
v_{x}+\left(\frac{1}{2} v^{2}+C \sigma\right)_{t}=0
$$




$$
\sigma_{x}+\left(\sigma v-\mathcal{R} v_{t t}\right)_{t}=0
$$

The second of these equations represents momentum conservation. As discussed in Appendix A, these two equations imply other conservation laws. I conjecture that there are an infinite number. The next of these, in its simplest form, is

$$
(\sigma v)_{x}+\left(\sigma v^{2}+\frac{1}{2} C \sigma^{2}+\mathcal{R}\left(\frac{1}{2} v_{t}^{2}-v v_{t t}\right)\right)_{t}=0
$$

Alternative forms can be obtained by adding a total $x$ derivative to the density and subtracting the corresponding $t$ derivative from the flux. In this way one can obtain an energy-conservation equation whose energy flux coincides with the momentum density:

$$
\left(\sigma v-\mathcal{R} v_{t t}\right)_{x}+\left(\sigma v^{2}+\frac{1}{2} C \sigma^{2}-\mathcal{R}\left(\frac{1}{2} v_{t}^{2}+2 v v_{t t}+C \sigma_{t t}\right)\right)_{t}=0
$$

It appears that the constant $\mathcal{R}$ must be positive, so that the new $v_{t t t}$ term in (8.64) has the effect of smoothing out the discontinuity in $v$ and the corresponding "corner" in $\sigma$ in the solution shown in Fig. 4 [29].

Now, in analogy with Table 1, one has a family of four equation systems; see Table 2. The pattern is somewhat different from Table 1, where the mass-conservation equation $\rho_{t}+(\rho v)_{x}=0$ held in each case. Its $x \leftrightarrow t$ analog in Table 2 (which is not mass conservation, but momentum conservation) becomes modified in the higher-derivative cases in the bottom half of the table.

\begin{tabular}{|l|l|}
\hline $\mathbf{R I F}$ & Higgs-vacuum hydrodynamics \\
$v_{x}+\left(\frac{1}{2} v^{2}\right)_{t}=0$ & $v_{x}+\left(\frac{1}{2} v^{2}+C \sigma\right)_{t}=0$ \\
$\sigma_{x}+(\sigma v)_{t}=0$ & $\sigma_{x}+(\sigma v)_{t}=0$ \\
\hline Schrödinger analog & GP analog \\
$v_{x}+\left(\frac{1}{2} v^{2}\right)_{t}=0$ & $v_{x}+\left(\frac{1}{2} v^{2}+C \sigma\right)_{t}=0$ \\
$\sigma_{x}+\left(\sigma v-\mathcal{R} v_{t t}\right)_{t}=0$ & $\sigma_{x}+\left(\sigma v-\mathcal{R} v_{t t}\right)_{t}=0$ \\
\hline
\end{tabular}

Table 2: Family of four equation-systems related to Higgs-vacuum hydrodynamics.

The simplest case (top left in Table 2) is denoted "RIF" for "relativistic incompressible fluid" since it corresponds to zero compressibility, $C=0$. Its Euler equation $v_{x}+v v_{t}=0$ is $\partial_{\mu} u^{\mu}=0$, the relativistic version of the usual incompressible-fluid condition $\vec{\nabla} \cdot \overrightarrow{\mathbf{v}}=0$ 4. The higher derivative cases, for lack of better names, are denoted as "Schrödinger analog" and "GP analog," even though the nature of the analogy is not clear. 
I suspect that in the "Schrödinger analog" case there is some function $\Psi(\sigma, \varphi)$, with $\varphi_{t}=v$, that satisfies some linear equation, but I have been unable to find this function and equation. I have found, though, that

$$
\sigma=-\frac{\mathcal{R}}{2}\left(\frac{v_{t t}}{v_{t}}\right)_{t}
$$

yields a solution to the $\sigma$ equation of the "Schrödinger analog" system. More generally one can add to $\sigma$ a term proportional to $v_{t}$, since this is a solution in the RIF case. (Cf. 27, 28.)

Introducing a velocity potential, $\varphi$, with $\varphi_{t}=v$, in the "GP analog" system allows one to solve the first equation for $\sigma$ :

$$
C \sigma=-\left(\varphi_{x}+\frac{1}{2} \varphi_{t}^{2}\right)
$$

One may then use this to eliminate $\sigma$ in the second equation to obtain

$$
\varphi_{x x}+2 \varphi_{t} \varphi_{t x}+\varphi_{t t}\left(\frac{3}{2} \varphi_{t}^{2}+\varphi_{x}\right)+\mathcal{R} C \varphi_{t t t t}=0
$$

Note that only the product of $\mathcal{R}$ and $C$ appears in this equation.

\section{9 'Bright' and 'dark' solitons}

For the GP equation it is well known that in the negative $g$ case, corresponding to attractive particle interactions, there are "bright soliton" solutions where the density has a $\mathrm{sech}^{2}$ form [25]. (Such solitons have been observed experimentally [30].) Remarkably, there are similar solutions to the "GP analog" equations, 8.63 8.64) if $\mathcal{R} C$ is negative. Although this "wrong sign" case probably has no physical significance [29] these solutions are of some interest because (i) they give support to the conjecture of integrability, and (ii) they hint at a symmetry, more subtle than $x \leftrightarrow t$, between the GP and "GP analog" equations.

To begin, one postulates a travelling-wave solution

$$
v=v(x-a t), \quad \sigma=\sigma(x-a t),
$$

where the constant $a$ represents the soliton's velocity. Substituting in Eq. (88.63) and integrating with respect to the variable $s \equiv x-$ at gives

$$
v-a\left(\frac{1}{2} v^{2}+C \sigma\right)=\text { const. } \equiv \frac{\left(1-\xi^{2}\right)}{2 a} .
$$


The constant of integration has been written in this form for later convenience. Note that when $\sigma \rightarrow 0$ the velocity $v$ tends to one or other of the constant values $v_{ \pm} \equiv(1 \pm \xi) / a$. Substituting Eq. (9.70) into Eq. (8.64) one obtains

$$
\sigma(1-a v)=-\mathcal{R} a^{3} v^{\prime \prime}
$$

where the constant of integration must be zero to have $v^{\prime \prime}=0$ when $\sigma=0$. Substituting for $\sigma$ from the previous equation leads to

$$
\left(\xi^{2}-(1-a v)^{2}\right)(1-a v)=-2 \mathcal{R} C a^{5} v^{\prime \prime} .
$$

Multiplying through by $a v^{\prime}$ and integrating yields

$$
4 \mathcal{R} C a^{6} v^{\prime 2}=a v(2-a v)\left(2\left(1-\xi^{2}\right)-2 a v+a^{2} v^{2}\right)+\text { const.. }
$$

The constant of integration here must be $-\left(1-\xi^{2}\right)^{2}$ so that $v^{\prime} \rightarrow 0$ when $v \rightarrow v_{ \pm}=$ $(1 \pm \xi) / a$. The result then simplifies to

$$
v^{\prime 2}=-\frac{1}{4 \mathcal{R} C a^{6}}\left((1-a v)^{2}-\xi^{2}\right)^{2} .
$$

Since the left-hand side is positive, a solution of this type is possible only if $\mathcal{R} C$ is negative. It is convenient to define

$$
\kappa \equiv \sqrt{-4 \mathcal{R} C} .
$$

Taking the square root of Eq. (9.75) and integrating yields the solution

$$
v=\frac{1}{a}\left[1 \pm \xi \tanh \left(\frac{\xi}{\kappa a^{2}}(x-a t)\right)\right] .
$$

From Eq. (9.71) one obtains the corresponding form of $\sigma$ :

$$
\sigma=\frac{\xi^{2}}{2 C a^{2}} \operatorname{sech}^{2}\left(\frac{\xi}{\kappa a^{2}}(x-a t)\right) .
$$

The above solution is characterized by two parameters $\xi$ and $a$ and by the sign choice, \pm , in Eq. (9.77). Each requires a little discussion. (i) Without loss of generality $\xi$ may be taken as positive. For $\xi=0$ the solution becomes trivial $(\sigma=0$ and $v=$ constant, representing the vacuum itself). For a single soliton one could set $\xi=1$ by choosing a frame of reference in which $v_{-}=0$. However, for the multi-soliton case, discussed below, the extra generality of the $\xi$ parameter is important. (ii) The $a$ parameter can have either sign but, for the NFA to be valid, the flow velocity must be small everywhere and hence $1 /|a| \ll 1$. Hence, the soliton moves at a hugely superluminal speed $|a| \gg 1$. 
The total momentum and energy carried by the soliton are, however, small and inversely proportional to its speed and speed-squared, respectively:

$$
P_{\text {soliton }}=\mathcal{M}\left(\frac{1}{a}\right), \quad E_{\text {soliton }}=\mathcal{M}\left(\frac{1}{a}\right)^{2}
$$

with

$$
\mathcal{M} \equiv \int_{-\infty}^{\infty} d x \sigma=\xi \frac{\kappa}{C}
$$

These results follow from evaluating the momentum and energy densities, which turn out to have the same $\operatorname{sech}^{2}$ form as $\sigma$; that is, using Eqs. (9.77, 9.78), one finds that

$$
\begin{gathered}
\sigma v-\mathcal{R} v_{t t}=\sigma / a \\
\sigma v^{2}+\frac{1}{2} C \sigma^{2}-\mathcal{R}\left(\frac{1}{2} v_{t}^{2}+2 v v_{t t}+C \sigma_{t t}\right)=\sigma / a^{2} .
\end{gathered}
$$

(iii) Another important property of the solution is that

$$
\sigma=\mp \frac{\kappa}{2 C} v_{t}
$$

with the upper/lower sign determined by the upper/lower sign in (9.77). I shall refer to the lower-sign choice as "solitons" and the upper-sign choice as "anti-solitons." Solitons have positive $v_{t}$ while anti-solitons have negative $v_{t}$.

Multi-soliton solutions can be constructed by using the ansatz $\sigma=\frac{\kappa}{2 C} v_{t}$. Both of the "GP analog" equations are then satisfied if

$$
v_{x}+v v_{t}=-\frac{\kappa}{2} v_{t t}
$$

which is the viscous Burgers' equation (2.12) with $x \leftrightarrow t$. This equation can be solved by the Hopf-Cole transformation [10]. One defines

$$
\psi=e^{\varphi / \kappa}, \quad \text { with } \quad \varphi_{t}=v,
$$

and requires $\psi$ to satisfy the linear equation

$$
\psi_{x}=-\frac{\kappa}{2} \psi_{t t}
$$

(the diffusion equation with $x \leftrightarrow t$ ). The resulting $v$ will then satisfy (9.84). The singlesoliton solution, 9.77 9.78) with the lower-sign choice, satisfies the $\sigma=\frac{\kappa}{2 C} v_{t}$ ansatz and by integrating $v$ one can find its corresponding $\varphi$. The appropriate constant of integration is a function of $x$ chosen so that $\psi=e^{\varphi / \kappa}$ satisfies Eq. (9.86). Hence, one obtains the 
$\psi$ function corresponding to a single bright soliton. By superposition, one can then write down a general class of solutions:

$$
\psi=\sum_{n} K_{n} \exp \left(\frac{t}{\kappa a_{n}}\right) \exp \left(-\left(x-x_{n}\right) \frac{\left(1+\xi_{n}^{2}\right)}{2 \kappa a_{n}^{2}}\right) \cosh \left(\frac{\xi_{n}}{\kappa a_{n}^{2}}\left(x-x_{n}-a_{n} t\right)\right),
$$

where $K_{n}, a_{n}, \xi_{n}$, and $x_{n}$ are arbitrary constants. By differentiation one can then obtain $v$ and $\sigma$ as

$$
v=\kappa \frac{\psi_{t}}{\psi}, \quad \sigma=\frac{\kappa^{2}}{2 C}\left(\frac{\psi_{t}}{\psi}\right)_{t} .
$$

Multi-anti-soliton solutions can be constructed in the same way by reversing the sign of $\kappa$ in the above. However, it is not clear if one can analytically construct solutions involving both solitons and anti-solitons.

As noted earlier, these 'bright soliton' solutions are probably unphysical since they require $\mathcal{R} C<0$ [29]. In the physical case, $\mathcal{R} C>0$, there are analogs of the 'dark soliton' solutions to the positive- $g$ GP equation. One may find these by the same method as before, but in Eq. (9.74) one now needs a different constant of integration. Choosing it to be $2 \xi^{2}-1$ yields a solution where $v$ tends asymptotically to $1 / a$ at $\pm \infty$. The resulting solution is

$$
\begin{gathered}
v=\frac{1}{a}\left[1 \pm \sqrt{4 a^{2} C \sigma_{0}} \operatorname{sech}\left(\sqrt{\frac{\sigma_{0}}{a^{2} \mathcal{R}}}(x-a t)\right)\right], \\
\sigma=\sigma_{0}\left[1-2 \operatorname{sech}^{2}\left(\sqrt{\frac{\sigma_{0}}{a^{2} \mathcal{R}}}(x-a t)\right)\right],
\end{gathered}
$$

where $\sigma_{0} \equiv \xi^{2} /\left(2 a^{2} C\right)$ is an arbitrary constant. In this solution both $v$ and $\sigma$ are constant at $\pm \infty$ but have a localized "dip" or "bump" that moves with velocity $a$. The dip/bump in $v$ can be arbitrarily small, but $\sigma$ has a dip that always goes negative, with a minimum value $-\sigma_{0}$. (See footnote [12] for remarks on negative $\sigma$.)

Since this 'dark soliton' solution does not have $\sigma \rightarrow 0$ at $\pm \infty$ it does not represent a perturbation of the vacuum. However, one can regard it as describing a localized disturbance in a larger, and much more slowly varying, perturbation of the vacuum. Thus, a solution like that shown in Fig. 4, for example, could be "decorated" with one or more 'dark soliton' dips. Calculating the momentum and energy densities for the dark soliton one finds, after subtracting the background values associated with $\sigma=\sigma_{0}$ and $v=1 / a$, that they are $\left(\sigma-\sigma_{0}\right) / a$ and $\left(\sigma-\sigma_{0}\right) / a^{2}$, respectively.

Note that the above solution can always be boosted to another frame with an "antiGalilean" transformation to obtain another solution. In particular one can boost to a frame where $v \rightarrow 0$ asymptotically. In that frame $v$ and $\sigma$ become functions of $t$ only. 


\section{Discussion}

There are two main points that I wish to emphasize: (i) Hydrodynamics in the emptyvacuum case makes perfect sense and describes an experimentally observed phenomenon, the free expansion of an atomic Bose-Einstein condensate when the atom-trap potential is turned off. (ii) Hydrodynamics in the Higgs-vacuum case gives very strange and exciting behaviour as a consequence of the fact that the speed of sound in the Higgs vacuum is formally infinite. The Higgs vacuum is a medium that is both ultrarelativistic (pressure $\gg$ energy density) and ultra-quantum, being a Bose-Einstein condensate with almost all its particles in the same quantum state. Not surprisingly, perhaps, its properties are very different from those of familiar media.

The vastly superluminal speed of hydrodynamic disturbances in the Higgs case will obviously require careful study. It may be important to point out that this phenomenon can occur without any material particle moving faster than the speed of light; see Fig. 6. In fact, a similar issue arises even for a non-relativistic Bose-Einstein condensate [31]: phonons with low momentum, $k \rightarrow 0$, travel at a finite speed of sound, yet they are made up of atoms that move with very small velocity $k / m$. In the non-relativistic case a spacetime picture like Fig. 6 predicts that the number of excited atoms present at any one time equals the ratio of the speed of sound to the speed of the individual atoms, a result that indeed follows from the usual Bogoliubov theory. See Ref. 31] for a detailed discussion of this point.

There is an apparent dichotomy between hydrodynamics, which implies "soft" modes that can be excited with arbitrarily little energy, and quantum field theory which (unless it explicitly contains massless particles) predicts an energy gap. In the empty-vacuum case the atoms carry a conserved quantum number, so there is a superselection rule fixing the total number of atoms. The theory splits into separate, non-communicating "sectors," each with a different total number of atoms. The vacuum, strictly speaking, is in the zero-atom sector and its lowest excitation, an atom/anti-atom pair, requires an energy $2 M c^{2}$, where $M$ is the mass of the atom. However, if one is given a system with $N$ atoms then the relevant "vacuum" state - the lowest energy state with $N$ atoms - has those atoms existing but dispersed in all directions to infinity (assuming a repulsive interaction $g>0$ ). This "vacuum" can be perturbed by bringing some or all of these atoms to finite distances from each other, and this may be done with arbitrarily little energy. In this sense, there are soft, hydrodynamic modes.

The situation for the Higgs vacuum is different. No conserved quantum number is 


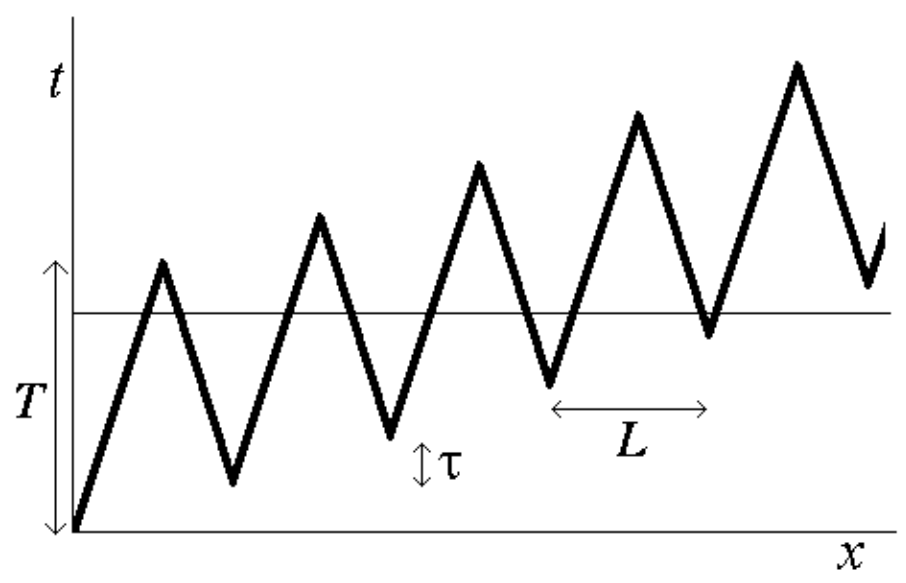

Figure 6: Possible spacetime picture of a superluminal phonon-like excitation. There are multiple pair-creation and pair-annihilation events organized so that the collective motion is fast $L / \tau \gg c$ while the individual particles move slowly $(L /(2 T)<c)$.

carried by the particles that spontaneously condense (the quanta of the scalar field that acquires a vacuum expectation value). According to conventional theory, the only excitations are massive (quasi)particles, and hence the system has a gap. However, the equation determining the inverse propagator at zero momentum actually has two solutions; $M_{h}^{2}$, where $M_{h}$ is the Higgs mass, and zero [32. This fact suggests that there may be some deeper, underlying theory that possesses gap-less, hydrodynamic modes. In the limit where this theory reduces to the conventional field theory, the realm of these hydrodynamic modes would shrink to $p^{\mu}=0$. Studying the hydrodynamic equations is then, I believe, a window into this unknown, deeper theory.

An example of what I have in mind is provided by the sine-Gordon equation 33 ]

$$
\frac{\partial^{2} \theta}{\partial t^{2}}-\frac{\partial^{2} \theta}{\partial x^{2}}+\sin \theta=0
$$

For small $\theta$ this equation reduces to the Klein-Gordon equation and hence the dispersion relation is $\omega(k)=\sqrt{1+k^{2}}$. One might therefore think that the spectrum of excitations necessarily has a gap. However, the non-linear equation possesses "breather" solutions

$$
\theta(x, t)=4 \arctan \left(\frac{\epsilon \sin \left(t / \sqrt{1+\epsilon^{2}}\right)}{\cosh \left(\epsilon\left(x-x_{0}\right) / \sqrt{1+\epsilon^{2}}\right)}\right),
$$

whose fundamental frequency, $\omega_{b}=1 / \sqrt{1+\epsilon^{2}}<1$ lies in the gap. (The sine-Gordon "breather" is unique in being stable. Similar breathers exist in the $\phi^{4}$ case but they decay 
due to $e^{-1 / \epsilon}$ couplings to linear, plane-wave modes [34.) The moral is that even if the linearized theory has only massive excitations, one cannot discount the possibility of soft, non-linear modes.

In this paper only the classical hydrodynamical equations have been considered. A task for the future is to re-cast the equations into Hamiltonian form so that one can apply canonical quantization. For a normal medium in the linear, acoustic regime this procedure would take one from classical sound waves to phonon quanta. For the vacuum case the quantization will be inherently non-linear.

Acknowledgements I thank Maurizio Consoli for discussions. This work was supported in part by the U.S. Department of Energy under Grant No. DE-FG05-97ER41031. 


\section{Appendix A: Towers of conservation laws}

For each of the $1+1$ dimensional equation systems in Table 1 there is an infinite tower of conservation laws

$$
D_{t}^{(n)}+F_{x}^{(n)}=0
$$

where $D$ is a density and $F$ is the associated flux. Each $D^{(n)}$ will be normalized such that its $\rho v^{n-1}$ term has unit coefficient. For $n=1,2,3$ the conserved quantities $\left(\int d x D^{(n)}\right)$ are mass, momentum, and twice the nonrelativistic energy. Of course, one has the freedom to add a total $x$-derivative to a density; i.e., $D^{\prime}=D+\Gamma_{x}$ and $F^{\prime}=F-\Gamma_{t}$ satisfy $D^{\prime}{ }_{t}+F^{\prime}{ }_{x}=0$.

The Burgers case is trivial, since it corresponds to non-interacting classical particles. One has simply

$$
D^{(n)}=\rho v^{n-1}, \quad F^{(n)}=\rho v^{n} .
$$

That is, not only are mass, momentum, and (nonrelativistic) energy (integrals of $\rho, \rho v$, and $\frac{1}{2} \rho v^{2}$ ) conserved, but - since $v$ is constant for a free particle — so is the integral of $\rho f(v)$ for any function of $v$. (Thus, the set of conservation laws above is really only a subset of a continuous infinity of conservation laws. I focus on this subset because it generalizes to the other equation systems.)

The empty-vacuum hydrodynamic equations include the effects of particle interactions (repulsive for $g>0$ ). The $D$ 's and F's are now polynomials in $\rho$ and $v$ :

$$
D^{(n)}=v^{n+1} d_{n}\left(g \rho / v^{2}\right) / g, \quad F^{(n)}=v^{n+2} f_{n}\left(g \rho / v^{2}\right) / g
$$

where the functions $d_{n}(s)$ and $f_{n}(s)$ (with $s=g \rho / v^{2}$ ) satisfy

$$
\begin{gathered}
(n+1) d_{n}=f_{n}^{\prime}-(1-2 s) d_{n}^{\prime}, \\
(n+2) f_{n}=(1+2 s) f_{n}^{\prime}-(1-s) d_{n}^{\prime},
\end{gathered}
$$

whose polynomial solutions are

$$
\begin{gathered}
d_{n}(s)=\sum_{i=1}^{[(n+1) / 2]} \frac{(n-1) !}{(n-2 i+1) !} \frac{1}{i !(i-1) !} s^{i}, \\
f_{n}(s)=\sum_{i=1}^{[(n+2) / 2]}(n+1-i) \frac{(n-1) !}{(n-2 i+2) !} \frac{1}{i !(i-1) !} s^{i} .
\end{gathered}
$$

Two noteworthy relations are

$$
D^{(n)}=\frac{1}{n} \frac{\partial D^{(n+1)}}{\partial v}
$$




$$
F^{(n)}=\frac{1}{2}\left(D^{(n+1)}+v D^{(n)}\right) .
$$

In the Schrödinger case one goes back to free particles, but now they are quantum mechanical. The D's and F's now involve higher derivatives. They can be expressed compactly in terms of the wavefunction $\psi$. Defining $\psi_{[N]}$ as the $N$ th derivative $\psi_{x x \ldots x}$ one has

$$
\begin{gathered}
D^{(2 N+1)}=\psi_{[N]}^{*} \psi_{[N]}, \\
F^{(2 N+1)}=D^{(2 N+2)}=\frac{-i}{2}\left(\psi_{[N]}^{*} \psi_{[N+1]}-\psi_{[N+1]}^{*} \psi_{[N]}\right), \\
F^{(2 N)}=\frac{1}{4}\left(2 \psi_{[N]}^{*} \psi_{[N]}-\psi_{[N-1]}^{*} \psi_{[N+1]}-\psi_{[N+1]}^{*} \psi_{[N-1]}\right) .
\end{gathered}
$$

By substituting $\psi=\sqrt{\rho / m} e^{i \varphi}$ with $v=\frac{\hbar}{m} \varphi_{x}$ one can obtain the $D$ 's and $F$ 's in terms of $\rho, v$, and their derivatives, though the expressions quickly become cumbersome. (See Table 3 with $g=0$ for the first few.) There is a straightforward generalization of these results to $d+1$ dimensions, with the $D^{(n)}$ 's being alternately scalar and vector densities and the $F^{(n)}$ 's correspondingly vector and tensor fluxes. I have not seen these conservation laws for the free Schrödinger equation in any quantum-mechanics textbook, although they surely must be known.

In the Gross-Pitaevskii (GP) case one has both quantum effects and interactions. It is a highly non-trivial property of this equation, related to its integrability, that it admits an infinite number of conservation laws in the $1+1$ dimensional case [35]. The first four of these are given explicitly in Table 3 .

\begin{tabular}{|l|l|l|}
\hline$n$ & $D^{(n)}$ & $F^{(n)}$ \\
\hline 1 & $\rho$ & $\rho v$ \\
\hline 2 & $\rho v$ & $\rho v^{2}+\frac{1}{2} g \rho^{2}-\mathcal{A}\left(\rho_{x x}-\frac{\rho_{x}^{2}}{\rho}\right)$ \\
\hline 3 & $\rho v^{2}+g \rho^{2}+\mathcal{A} \frac{\rho_{x}^{2}}{\rho}$ & $\rho v^{3}+2 g \rho^{2} v+\mathcal{A}\left(\frac{3 \rho_{x}^{2} v}{\rho}+2 \rho_{x} v_{x}-2 \rho_{x x} v\right)$ \\
\hline 4 & $\rho v^{3}+3 g \rho^{2} v$ & $\rho v^{4}+\frac{9}{2} g \rho^{2} v^{2}+g^{2} \rho^{3}$ \\
& $+\mathcal{A}\left(\frac{3 \rho_{x}^{2} v}{\rho}+2 \rho_{x} v_{x}-2 \rho_{x x} v\right)$ & $+\mathcal{A}\left(-5 \rho_{x x} v^{2}+6 \frac{\rho_{x}^{2}}{\rho} v^{2}+4 \rho_{x} v v_{x}+2 \rho v_{x}^{2}-2 \rho v v_{x x}\right)$ \\
& & $\mathcal{A} g\left(\frac{9}{2} \rho_{x}^{2}-3 \rho \rho_{x x}\right)$ \\
& & $+\mathcal{A}^{2}\left(\frac{-2 \rho_{x x x} \rho_{x}}{\rho}+\frac{2 \rho_{x x}^{2}}{\rho}+\frac{\rho_{x x} \rho_{x}^{2}}{\rho^{2}}-\frac{\rho_{x}^{4}}{\rho^{3}}\right)$ \\
\hline
\end{tabular}

Table 3: The first four conservation laws, $D_{t}+F_{x}=0$, for the GP equation system. $\mathcal{A}=\hbar^{2} /\left(4 m^{2}\right) . D^{(1)}$ is the mass density, $D^{(2)}$ is the momentum density, and $D^{(3)}$ is twice the nonrelativistic energy density. 
I turn now to the equation systems of Table 2. The RIF and Higgs-vacuum hydrodynamics cases are related by $x \leftrightarrow t$ to the Burgers and empty-vacuum cases, respectively and so inherit the same set of conservation laws as

$$
D_{x}^{(n)}+F_{t}^{(n)}=0
$$

(Note that I choose not to switch the names $D$ and $F$, so one needs to remember that now $D$ is a flux and $F$ is a density.)

For the "Schrödinger analog" case $(\mathcal{R} \neq 0$, but $C=0)$ it is easily verified that there are an infinite number of conservation laws that need only involve $\sigma, v, v_{t}, v_{t t}$ :

$$
\begin{gathered}
F_{n}=\sigma v^{n}+\frac{1}{2} \mathcal{R}\left((n-1)^{2} v^{n-2} v_{t}^{2}-2 v^{n-1} v_{t t}\right), \\
D_{n}=\sigma v^{n-1}+\frac{1}{2} \mathcal{R}(n-1)(n-2) v^{n-3} v_{t}^{2}
\end{gathered}
$$

(One may of course modify these by adding a total $t$-derivative to $D$ and subtracting the corresponding $x$-derivative from $F$. I have chosen to do this for the $n=2$ case in Table 4 in order for $D^{(2)}$, interpreted as the energy flux, to equal the momentum density $F^{(1)}$.)

Table 4 shows the first four conservation laws for the "GP analog" system.

\begin{tabular}{|l|l|l|}
\hline$n$ & $D^{(n)}$ & $F^{(n)}$ \\
\hline 1 & $\sigma$ & $\sigma v-\mathcal{R} v_{t t}$ \\
\hline 2 & $\sigma v-\mathcal{R} v_{t t}$ & $\sigma v^{2}+\frac{1}{2} C \sigma^{2}-\mathcal{R}\left(\frac{1}{2} v_{t}^{2}+2 v v_{t t}+C \sigma_{t t}\right)$ \\
\hline 3 & $\sigma v^{2}+C \sigma^{2}+\mathcal{R} v_{t}^{2}$ & $\sigma v^{3}+2 C \sigma^{2} v+\mathcal{R}\left(2 v v_{t}^{2}-v^{2} v_{t t}+2 C\left(\sigma_{t} v_{t}-\sigma v_{t t}\right)\right)$ \\
\hline 4 & $\sigma v^{3}+3 C \sigma^{2} v$ & $\sigma v^{4}+\frac{9}{2} C \sigma^{2} v^{2}+C^{2} \sigma^{3}+\mathcal{R}\left(\frac{9}{2} v^{2} v_{t}^{2}-v^{3} v_{t t}\right)$ \\
& $+\mathcal{R}\left(3 v v_{t}^{2}-4 C \sigma v_{t t}\right)$ & $+\mathcal{R} C\left(6 \sigma_{t} v v_{t}-3 \sigma v_{t}^{2}-10 \sigma v v_{t t}\right)$ \\
& & $+2 \mathcal{R}^{2} C v_{t t}^{2}+\mathcal{R} C^{2}\left(2 \sigma_{t}^{2}-4 \sigma \sigma_{t t}\right)$ \\
\hline
\end{tabular}

Table 4: The first four conservation laws, $D_{x}+F_{t}=0$, for the "GP analog" system (Higgs-vacuum hydrodynamics plus higher-derivative corrections). $F^{(1)}$ is the momentum density and $F^{(2)}$ is the energy density. 


\section{References}

[1] G. 't Hooft, In Search of the Ultimate Building Blocks (Cambridge University Press, 1997) p. 70.

[2] A particle-language description of the Higgs vacuum is discussed in M. Consoli and P. M. Stevenson, Int. J. Mod. Phys A 15, 133 (2000). (The word "phonon" is used inappropriately in this reference and should be replaced by the term "quasiparticle.") The Higgs boson in this picture is a "roton-like" quasiparticle; see M. Consoli and E. Costanzo, Eur. Phys. J. C 33, 297 (2004).

[3] R. Jackiw, V. P. Nair, S.-Y. Pi, and A. P. Polychronakos hep-ph/0407101.

[4] L. D. Landau and E. M. Lifshitz, Fluid Mechanics, Vol. 6 of Course of Theoretical Physics (Pergamon Press, Oxford, 1959); E. M. Lifschitz and L. P. Pitaevskii, Statistical Physics, Part 2 (Pergamon Press, London, 1980).

[5] R. Courant and K. O. Friedrichs, Supersonic Flow and Shock Waves (Interscience Publishers, 1948) (reprinted Springer-Verlag, 1976), Sect. 19.

[6] C. J. Chapman, High Speed Flow (Cambridge University Press, 2000).

[7] See Eqs. (1.2.62-66) of Ref. 3].

[8] L. C. Evans, Partial Differential Equations (American Mathematical Society, 1998).

[9] See, for example, K. Huang, Statistical Mechanics, 2nd ed. (John Wiley, New York, 1987).

[10] See, for example, J. V. José and E. J. Salatan, Classical Dynamics: a contemporary approach (Cambridge Univ. Press, 1998) Sec. 9.5.2.

[11] $g$ may be negative, but then higher-order terms in $\mathcal{E}$ are crucial to ultimate stability.

[12] Convexification of the effective potential (see, e.g., ref [13]) is an issue if $\sigma$ goes negative; i.e., $n<n_{v}$. Convexification implies that for negative $\sigma$ the energy density should really be flat, $\mathcal{E}=0$. However, if the timescale involved is not enormously long the expression $\mathcal{E}=\frac{1}{2} C \sigma^{2}$ should still be appropriate in a metastable sense, akin to briefly supercooling a system below its thermodynamic phase transition. This issue does not arise for the solution in Section 6 , which has $\sigma \geq 0$ everywhere. 
[13] M. E. Peskin and D. V. Schroeder, An introduction to quantum field theory, (AddisonWesley, 1995), p. 368.

[14] P. M. Stevenson, in Proceedings of the Second Meeting on CPT and Lorentz Symmetry, Ed. V. A. Kostelecký (World Scientific, Singapore, 2002). hep-ph/0109204.

[15] D. A. Kirshnitz and V. L. Polyachenko, Sov. Phys. JETP 19, 514 (1964)

[16] S. A. Bludman and M. A. Ruderman, Phys. Rev. 170, 1176 (1968); M. A. Ruderman, Phys. Rev. 172, 1286 (1968); S. A. Bludman and M. A. Ruderman, Phys. Rev. D 1, 3243 (1970).

[17] B. D. Keister and W. N. Polyzou, Phys. Rev. C 54, 2023 (1996).

[18] S. R. de Groot, W. A. van Leeuwen, and Ch. G. van Weert, Relativistic Kinetic Theory: Principles and Applications (North Holland, Amsterdam, 1980).

[19] Lax representations of polytropic systems in $1+1$ dimensions have been studied: A. Constandache, A. Das, and F. Toppan, Lett. Math. Phys. 60, 197 (2002) (hep-th/0110097); J. C. Brunelli and A. Das, Phys. Lett. A 235, 597 (1997) (solv-int/9706005).

[20] Note that although the flow is nonrelativistic, $v \ll 1$, it is not "slow" because $v$ is always much greater than the speed of sound, which is zero here.

[21] S. Stringari, Phys. Rev. Lett. 77, 2360 (1996).

[22] F. Dalfovo, C. Minniti, S. Stringari, and L. Pitaevskii, Phys. Lett. A 227, 259 (1997).

[23] M.-O. Mewes et al, Phys Rev. Lett. 77, 416 (1996); M. H. Anderson et al, Science 269, 198 (1995).

[24] It need not concern us here but in the atomic-physics case there are subtleties in how 3-dimensional physics reduces to effective 1-dimensional physics. See P. Pedri, L. Santos, P. Öhberg, and S. Stringari, cond-mat/0304660

[25] C. J. Pethick and H. Smith, Bose-Einstein Condensation in Dilute Gases (Cambridge Univ. Press, 2002).

[26] This substitution, in the Schrödinger-equation case $g=0$, is due to E. Madelung, Z. Phys. 40, 322 (1927). 
[27] Ph. Choquard, Found. Phys. 31, 623 (2001); Ph. Choquard and J. Wagner, Found. Phys. 32, 1225 (2002).

[28] The $\rho$ equation in the Burgers' case admits a more general solution, $\rho=f(v) v_{x}+$ $g(v)\left(v^{2}-2 v_{x} \varphi\right)$, where $f$ and $g$ are arbitrary functions and $\varphi_{x}=v$. However, only the case $f=$ constant and $g=0$ is consistent with Galilean invariance. In the relativistic case one would need $f \propto \gamma=1 / \sqrt{1-v^{2}}$ for Lorentz invariance.

[29] However, it is conceivable that $\mathcal{R}$ may be negative in the same sense that $g$ can be negative in the empty-vacuum case; see footnote [11.

[30] K. E. Strecker, G. B. Partridge, A. G. Truscott, and R. G. Hulet, Nature 417,150 (2002); New J. Phys. 5, 73 (2003).

[31] P. M. Stevenson, Phys. Rev. A 68, 055601 (2003) (cond-mat/0306372).

[32] M. Consoli, Phys. Rev. D 65, 105017 (2002).

[33] M. J. Ablowitz, D. J. Kaup, A. C. Newell, and H. Segur, Phys. Rev. Lett. 30, 1262 (1973)

[34] H. Segur and M. D. Kruskal, Phys. Rev. Lett. 58, 747 (1987).

[35] V. E. Zakharov and A. B. Shabat, Sov. Phys. JETP 3462 (1972). (Zh. Eksp. Teor. Fiz. 61, 118 (1971)) 